\title{
Correction: A New Quaternion-Based Kalman Filter for Real-Time Attitude Estimation Using the Two-Step Geometrically-Intuitive Correction Algorithm. Sensors 2017, 17, 2146
}

\author{
Kaiqiang Feng ${ }^{1,2}$, Jie Li ${ }^{1,2, *}$, Xiaoming Zhang ${ }^{1,2}$, Chong Shen ${ }^{1,2}$, Yu Bi ${ }^{1,2}$, Tao Zheng ${ }^{1,2}$ and \\ Jun Liu ${ }^{1,2}$ \\ 1 Key Laboratory of instrumentation Science \& Dynamic Measurement, Ministry of Education, \\ North University of China, Taiyuan 030051, China; b1506011@st.nuc.edu.cn (K.F.); \\ zxm_auto@nuc.edu.cn (X.Z.); shenchong@nuc.edu.cn (C.S.); b1506009@st.nuc.edu.cn (Y.B.); \\ s1506044@st.nuc.edu.cn (T.Z.); liuj@nuc.edu.cn (J.L.) \\ 2 National Key Laboratory for Electronic Measurement Technology, North University of China, \\ Taiyuan 030051, China \\ * Correspondence: Lijie@nuc.edu.cn; Tel.: +86-139-3454-7414
}

Received: 28 October 2017; Accepted: 31 October 2017; Published: 3 November 2017

The authors wish to make the following corrections to their paper [1]:

1. In page 7, "The two-step correction process is described as follows:" should be revised as "The two-step correction process is described as follows [31]:". Meanwhile, the corresponding reference "31. Del Rosario, M.B.; Lovell, N.H.; Redmond, S.J. Quaternion-based complementary filter for attitude determination of a smartphone. IEEE Sens. J. 2016, 16, 6008-6017." should be added in the references part.

2. In page 11, "and we can rewrite the function as:" should be revised as "and we can rewrite the function as [32]:". Meanwhile, the corresponding reference "32. Valenti, R.G.; Dryanovski, I.; Xiao, J.Z. A linear kalman filter for marg orientation estimation using the algebraic quaternion algorithm. IEEE Trans. Instrum. Meas. 2016, 65, 467-481." should be added in the references part.

The authors would like to apologize for any inconvenience caused to the readers by these changes.

Conflicts of Interest: The authors declare no conflict of interest.

\section{Reference}

1. Feng, K.; Li, J.; Zhang, X.; Shen, C.; Bi, Y.; Zheng, T.; Liu, J. A new quaternion-based kalman filter for real-time attitude estimation using the two-step geometrically-intuitive correction algorithm. Sensors 2017, 17, 2146. [CrossRef] [PubMed]

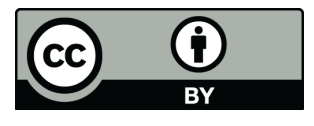

(c) 2017 by the authors. Licensee MDPI, Basel, Switzerland. This article is an open access article distributed under the terms and conditions of the Creative Commons Attribution (CC BY) license (http:/ / creativecommons.org/licenses/by/4.0/). 